\title{
Clinical Study \\ Incidence of Retinopathy of Prematurity in Extremely Premature Infants
}

\author{
Alparslan Şahin, Muhammed Şahin, Fatih Mehmet Türkcü, Abdullah Kürşat Cingü, \\ Harun Yüksel, Yasin Çınar, Şeyhmus Arı, and İhsan Çaça
}

Department of Ophthalmology, School of Medicine, Dicle University, Diyarbakır, Turkey

Correspondence should be addressed to Alparslan Şahin; dralparslansahin@gmail.com

Received 14 November 2013; Accepted 24 February 2014; Published 9 March 2014

Academic Editors: S. A. Sarker and K. Tokiwa

Copyright (C) 2014 Alparslan Şahin et al. This is an open access article distributed under the Creative Commons Attribution License, which permits unrestricted use, distribution, and reproduction in any medium, provided the original work is properly cited.

\begin{abstract}
Purpose. To investigate the incidence and the severity of retinopathy of prematurity (ROP) in extremely preterm infants born before 28 weeks of gestation in southeastern Turkey. Methods. A retrospective chart review was performed for infants born before 28 weeks of gestation. The following data were reviewed: gender, gestational age (GA), birth weight (BW), zone and stage of ROP, presence of plus disease, and treatment for ROP if needed. Infants were divided into 2 groups according to GA as follows: group 1 included infants of GAs 25 weeks and under; group 2 included infants of GAs less than 28 weeks and over 25 weeks. Results. The incidence of any ROP in the whole cohort, in group 1, and in group 2, was $66.0 \%, 95.5 \%$, and $58.6 \%$, respectively. Incidence of any ROP was significantly associated with BW and GA $(P=0.014$ and $P=0.002$, resp.). The overall incidence of type 1 ROP was $35.8 \%$ (59.1\% in group 1 and $29.9 \%$ in group 2). Development of type 1 ROP was independently associated with GA. Conclusion. Any ROP was significantly associated with BW and GA. Extremely premature infants with lower GA were found to be more likely to develop type 1 ROP. BW cannot predict the development of type 1 ROP.
\end{abstract}

\section{Introduction}

Retinopathy of prematurity (ROP) is an important cause of vision loss in children, especially in extremely premature infants [1]. The survival rates of extremely premature infants have been increased with the improvement in the neonatal intensive care technologies and increased availability of healthcare services in recent years [2].

Infants with gestational age (GA) less than 28 weeks have particularly more risk for the development of ROP in developed countries $[3,4]$. In developing countries, the incidence of ROP is rising with the improvement of the survival rates of extremely premature infants $[5,6]$. These infants are more tending to develop severe ROP and require treatment [7].

In the present study, we aimed to determine the incidence and the severity of ROP in extremely premature infants in southeast part of Turkey.

\section{Materials and Methods}

Dicle University Health Research Ethics Committee approved the study. The medical records of the premature infants examined between September 2010 and August 2012 in Retina Department of Dicle University Medical Faculty were retrospectively reviewed. The patients with a GA under 28 weeks were included in the study. The patients who died prior to retinal screening examination and had lack of relevant data in their medical records were excluded.

All patients were first examined using indirect ophthalmoscope at 31 weeks of GA and follow-up examinations were performed twice per week to every 3 weeks, depending on the zone and severity of the disease [8]. The pupil was dilated with 2.5\% phenylephrine (Mydfrin, Alcon, USA) and 0.5\% tropicamide (Tropamide, Bilim, Turkey). The infants were examined after instillation of a topical anesthesthetic, $0.5 \%$ proparacaine (Alcaine, Alcon, USA). A lid speculum and 
TABLE 1: Baseline characteristics of the study population.

\begin{tabular}{lccccc}
\hline Group & $n$ & Gender $(\mathrm{M} / \mathrm{F})$ & $\begin{array}{c}\text { GA (week) } \\
(\text { Mean } \pm \text { SD) }\end{array}$ & Range & $\begin{array}{c}\text { BW (gram) } \\
(\text { Mean } \pm \text { SD) }\end{array}$ \\
\hline 1 & 22 & $9 / 13$ & $24.41 \pm 0.8$ & $23-25$ & $825.5 \pm 211.4$ \\
2 & 87 & $42 / 45$ & $26.4 \pm 0.5$ & $26-27$ & $997.4 \pm 206.2$ \\
All & 109 & $51 / 58$ & $26.05 \pm 1.0$ & $23-27$ & $963.4 \pm 217.4$ \\
\hline
\end{tabular}

BW: birth weight; GA: gestational age; M: male; F: female.

TABLE 2: Distribution of the patients between the groups according to the stages of ROP.

\begin{tabular}{lccccccc}
\hline Group & No ROP & Any ROP & Stage 1 & Stage 2 & Stage 3 & AP-ROP & Type 1 ROP \\
\hline 1 & 1 & 21 & 4 & 8 & 5 & 4 & 13 \\
2 & 36 & 51 & 14 & 14 & 15 & 8 & 26 \\
All & 37 & 72 & 18 & 22 & 20 & 12 & 39 \\
\hline
\end{tabular}

ROP: retinopathy of prematurity.

scleral indentator were used to visualize the peripheral retina. All examinations were performed by the same ophthalmologist (A.Ş.) experienced in screening premature infants for ROP.

The following data were reviewed: gender, GA, birth weight (BW), zone and stage of ROP, presence of plus disease, and treatment for ROP if needed. The endpoints of data collection in the study defined as completely vascularization of the retina or ROP progressed to the stage require treatment according to ET-ROP recommendations [9].

The infants were divided into two groups on the basis of their GA. Group 1 included infants of GAs 25 weeks and under; group 2 included infants of GAs less than 28 weeks and over 25 weeks. The incidence of any ROP, type 1 ROP, by means of BW and GA, was also evaluated with respect to gender. The description of GA was used as defined by American Academy of Pediatrics [10]. Infants requiring treatment for ROP were defined as having onset of type 1 ROP as used in ETROP study [9]. Treatments were performed either with laser photocoagulation or intravitreal bevacizumab injection. Even though ETROP study recommended ablative laser photocoagulation in the treatment of prethreshold ROP, we preferred intravitreal bevacizumab (IVB) monotherapy since August 2011.

\section{Statistical Analysis}

Mann-Whitney $U$-test was used to compare continuous variables. A $P$ value of less than 0.05 was accepted as significant. A logistic regression analysis was used to evaluate the association of GA and BW with development of any ROP and type 1 ROP.

\section{Results}

From September 2010 to August 2012, the medical records of 526 premature infants were reviewed in the Hospital of Dicle University, and 109 of them were eligible for the study. 22 of the premature infants were $\leq 25$ weeks of GA (group 1) and 87 of them were over 25 weeks of GA (group 2). There were
51 male $(46.8 \%)$ and $48(53.2 \%)$ female infants. The mean BW in groups 1 and 2 was $825.5 \pm 211.4$ and $997.4 \pm 206.2$, respectively $(P<0.001)$. The mean GA in groups 1 and 2 was $24.41 \pm 0.8$ weeks and $26.46 \pm 0.5$ weeks, respectively $(P<0.001)$. The GA and the BW of the study population were summarized in Table 1 .

The incidence of any ROP was $66.0 \%$ (72 of 109 infants). The incidence of any ROP in groups 1 and 2 was $95.5 \%$ and $58.6 \%$, respectively $\left(P=0.001, \chi^{2}=11.15\right)$, with an odds ratio of 15.54 (95\% CI 1.99-120.78).

The overall incidence of type 1 ROP was 35.8\% (39 of 109 infants). Type 1 ROP incidence in group 1 and group 2 was $59.1 \%$ and $29.9 \%$, respectively $\left(P=0.011, \chi^{2}=6.51\right)$, with an odds ratio of 3.38 (95\% CI 1.29-8.90). Table 2 summarizes the incidence of the stages of ROP and type 1 ROP according to groups.

The mean GA of infants who had any ROP was $25.8 \pm 1.12$ weeks and the mean BW was $924.4 \pm 205.0$ grams. The mean GA of infants who did not have ROP was $26.4 \pm 0.6$ weeks $(P=0.002)$, and BW was $1036.2 \pm 223.8$ grams $(P=0.014)$. We did not find any significant difference with respect to the mean BW and GA between patients with and without type 1 ROP (Table 3).

GA was independently associated with any ROP $(P=$ $0.018)$ and type 1 ROP $(P=0.038)$. However BW was not independently associated with any ROP and type 1 ROP $(P=$ 0.178 and $P=0.714$, resp.).

With respect to gender, the comparisons of the incidence of any ROP, type 1 ROP, the mean BW, and the mean GA were not statistically significant $(P=0.75, P=0.76, P=0.22$, and $P=0.75$, resp.).

\section{Discussion}

The survival rate of extremely premature infants continuously increases as a consequence of the advancement of neonatal care [5]. These infants are at a particularly high risk for developing more severe ROP and they usually need treatment $[3,11]$. In developing countries, severe ROP incidence is also high in more mature infants [5]. On the other hand, there 
TABLE 3: The mean BW and GA in patients with and without type 1 ROP in the groups.

\begin{tabular}{|c|c|c|c|c|c|c|}
\hline \multirow{2}{*}{ Presence of type 1 ROP } & \multicolumn{3}{|c|}{ BW (gram) } & \multicolumn{3}{|c|}{ GA (week) } \\
\hline & Yes & No & $P$ value & Yes & No & $P$ value \\
\hline Group 1 & $826.7 \pm 225.7$ & $823.7 \pm 200.1$ & 0.828 & $24.31 \pm 0.8$ & $24.56 \pm 0.9$ & 0.307 \\
\hline Group 2 & $972.5 \pm 178.2$ & $1008.5 \pm 217.9$ & 0.335 & $26.42 \pm 0.5$ & $26.48 \pm 0.5$ & 0.656 \\
\hline All & $1014.0 \pm 220.8$ & $1007.0 \pm 206.4$ & 0.899 & $26.38 \pm 0.5$ & $26.48 \pm 0.5$ & 0.429 \\
\hline
\end{tabular}

BW: birth weight; GA: gestational age; ROP: retinopathy of prematurity.

is another risk for the development of the severe ROP is the increase of the survival rates of the extremely premature infants, which may affect the incidence and the severity of ROP in the developing countries [12].

In the current study, the overall incidence of any ROP was $66.0 \%$, which was lower than the $85 \%$ incidence reported in the ETROP study [13]. However, similarly, Isaza and Arora [4] reported the overall incidence of any ROP was $64.7 \%$, and Teed and Saunders [11] reported 71\%.

The overall incidence of any ROP was $95.5 \%$ in group 1 and $58.6 \%$ in group 2. Isaza and Arora found the incidence as $88 \%$ versus $48 \%$, respectively. Similarly, Teed and Saunders [11] reported the overall incidence of any ROP in the $<25$ weeks group and in the 25 to 27 weeks group as $87 \%$ versus $62 \%$, respectively.

On the other hand, the overall incidence of type 1 ROP was found to be higher in our study (35.8\%) than that of previously reported one. Teed and Saunders [11] reported $13 \%$ and Isaza and Arora [4] reported $11.6 \%$ of patients had type 1 ROP. Gilbert et al. reported that more mature infants were tending to develop severe ROP in less developed countries compared with developed countries [5]. They concluded that criteria of screening programs should be adjusted according to relevant population. Mutlu et al. reported that frequency of ROP in Turkey was similar to that in the United States; however, according to their results, the rate of severe ROP necessitating treatment seems to be higher in Turkey [14].

In the current study, the mean BWs in group 1 and group 2 were $825.5 \pm 211.4$ and $997.4 \pm 206.2$ grams, which were higher compared to the previously reported studies conducted in USA [11] and Canada [4]. The survival rates of the infants in newborn intensive care units in developing countries are lower than those of developed countries. We suggest that higher mean BW in our study, compared to developed countries, is the result of lower survival rates of extremely premature infants with relatively lower BW in Turkey. In Sweden, survival rate of extremely preterm infants born before 27 gestational weeks was $70 \%$ [15]. The survival rate of extremely premature infants (27 weeks and under) in our newborn intensive care unit was 39\%. The mean BW in

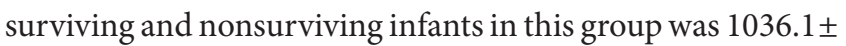
273 gr and $780 \pm 154$ gr, respectively (unpublished data).

We found that the development of type 1 ROP was associated with lower GA but not associated with BW. Woo et al. suggested that GA is a better predictor of ROP than BW according to their twin-paired study [16]. They concluded that maturity is more important in the pathogenesis of ROP than intrauterine growth. Teed and Saunders reported similar findings in their study in infants with a GA under 25 weeks.
They suggested that it was a unique characteristic of ROP in extremely premature infants and proposed that other neonatal risk factors may also have some additional effect in the severity and progression of ROP in such infants [11].

In conclusion, our findings suggested that development of any ROP was associated with both lower BW and lower GA. Furthermore, type 1 ROP development was independently associated with GA. On the other hand, no association was found between type $1 \mathrm{ROP}$ and BW in extremely preterm infants with a GA of less than 28 weeks. Although the incidence of any ROP was similar to that of previous reports, the incidence of type 1 ROP in our study population is higher.These results represent only the experience of single center in Turkey and may not be generalized to other different populations. Larger prospective, cohort studies are needed to clarify the relationship of type 1 ROP with BW and GA in extremely premature infants.

\section{Disclosure}

The authors have no proprietary or financial interest in the products mentioned in this study. The authors received no financial support for this study. This paper has not been published nor submitted simultaneously for publication elsewhere.

\section{Conflict of Interests}

The authors declare that there is no conflict of interests regarding the publication of this paper.

\section{References}

[1] C. Gilbert, "Retinopathy of prematurity: a global perspective of the epidemics, population of babies at risk and implications for control," Early Human Development, vol. 84, no. 2, pp. 77-82, 2008.

[2] D. J. Field, J. S. Dorling, B. N. Manktelow, and E. S. Draper, "Survival of extremely premature babies in a geographically defined population: prospective cohort study of 1994-1999 compared with 2000-2005," British Medical Journal, vol. 336, no. 7655, pp. 1221-1223, 2008.

[3] D. Austeng, K. B. M. Källen, U. W. Ewald, P. G. Jakobsson, and G. E. Holmström, "Incidence of retinopathy of prematurity in infants born before 27 weeks' gestation in Sweden," Archives of Ophthalmology, vol. 127, no. 10, pp. 1315-1319, 2009.

[4] G. Isaza and S. Arora, "Incidence and severity of retinopathy of prematurity in extremely premature infants," Canadian Journal of Ophthalmology, vol. 47, pp. 296-300, 2012. 
[5] C. Gilbert, A. Fielder, L. Gordillo et al., "Characteristics of infants with severe retinopathy of prematurity in countries with low, moderate, and high levels of development: implications for screening programs," Pediatrics, vol. 115, no. 5, pp. e518-e525, 2005.

[6] A. Sahin, M. Sahin, A. K. Cingü et al., "Intravitreal bevacizumab monotherapy for retinopathy of prematurity," Pediatrics International, vol. 55, no. 5, pp. 599-603, 2013.

[7] J. B. Fortes Filho, G. U. Eckert, L. Procianoy, C. K. Barros, and R. S. Procianoy, "Incidence and risk factors for retinopathy of prematurity in very low and in extremely low birth weight infants in a unit-based approach in southern Brazil," Eye, vol. 23, no. 1, pp. 25-30, 2009.

[8] American Academy of Pediatrics, "Screening examination of premature infants for retinopathy of prematurity," Pediatrics, vol. 117, pp. 572-576, 2006.

[9] W. V. Good, R. J. Hardy, V. Dobson et al., "Revised indications for the treatment of retinopathy of prematurity: results of the early treatment for retinopathy of prematurity randomized trial," Archives of Ophthalmology, vol. 121, no. 12, pp. 1684-1696, 2003.

[10] L. R. Blackmon, D. G. Batton, E. F. Bell et al., "Age terminology during the perinatal period," Pediatrics, vol. 114, no. 5, pp. 13621364, 2004.

[11] R. G. W. Teed and R. A. Saunders, "Retinopathy of prematurity in extremely premature infants," Journal of AAPOS, vol. 13, no. 4, pp. 370-373, 2009.

[12] D. E. Ballot, T. F. Chirwa, and P. A. Cooper, "Determinants of survival in very low birth weight neonates in a public sector hospital in Johannesburg," BMC Pediatrics, vol. 10, article 30, 2010.

[13] W. V. Good, "The incidence and course of retinopathy of prematurity: findings from the early treatment for retinopathy of prematurity study," Pediatrics, vol. 116, no. 1, pp. 15-23, 2005.

[14] F. M. Mutlu, H. I. Altinsoy, T. Mumcuoglo et al., "Screening for retinopathy of prematurity in a tertiary care newborn unit in Turkey: frequency, outcomes, and risk factor analysis," Journal of Pediatric Ophthalmology and Strabismus, vol. 45, no. 5, pp. 291-298, 2008.

[15] K. Maršál, V. Fellman, L. Hellström-Westas et al., "One-year survival of extremely preterm infants after active perinatal care in Sweden," Journal of the American Medical Association, vol. 301, no. 21, pp. 2225-2233, 2009.

[16] S. J. Woo, K. H. Park, J. Ahn et al., "A co-twin study of the relative effect of birth weight and gestational age on retinopathy of prematurity," Eye, vol. 25, no. 11, pp. 1478-1483, 2011. 


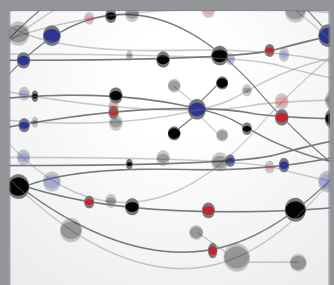

The Scientific World Journal
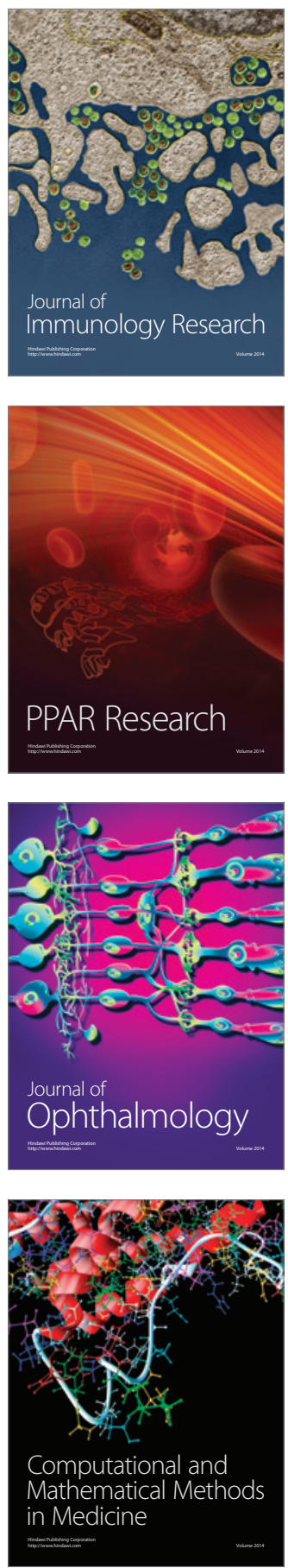

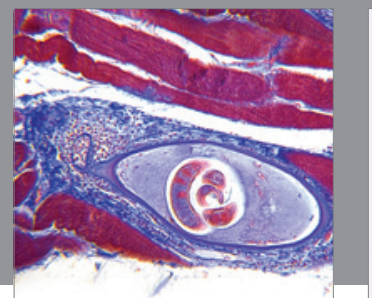

Gastroenterology

Research and Practice
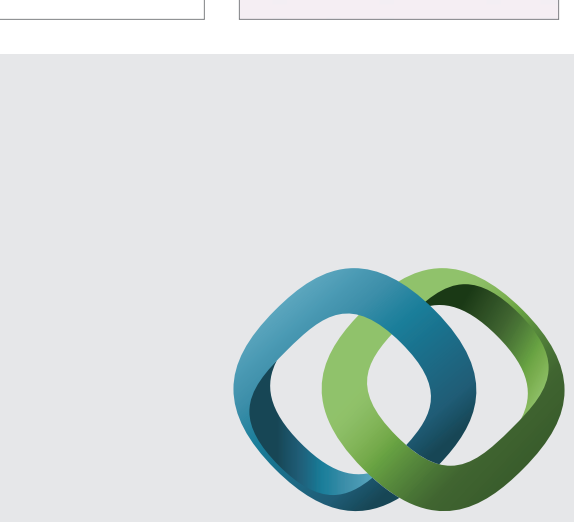

\section{Hindawi}

Submit your manuscripts at

http://www.hindawi.com
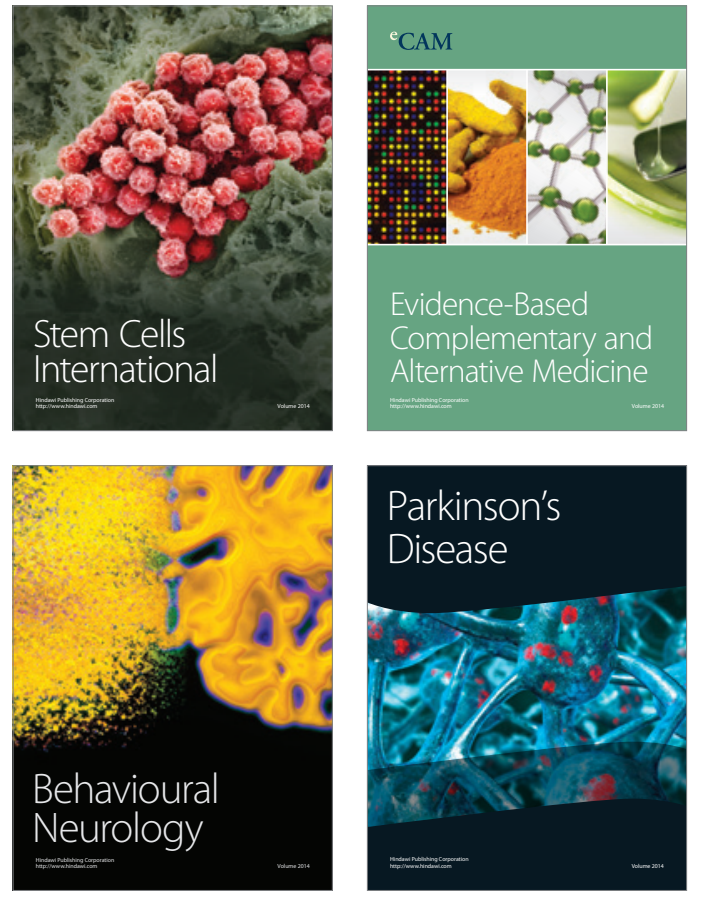
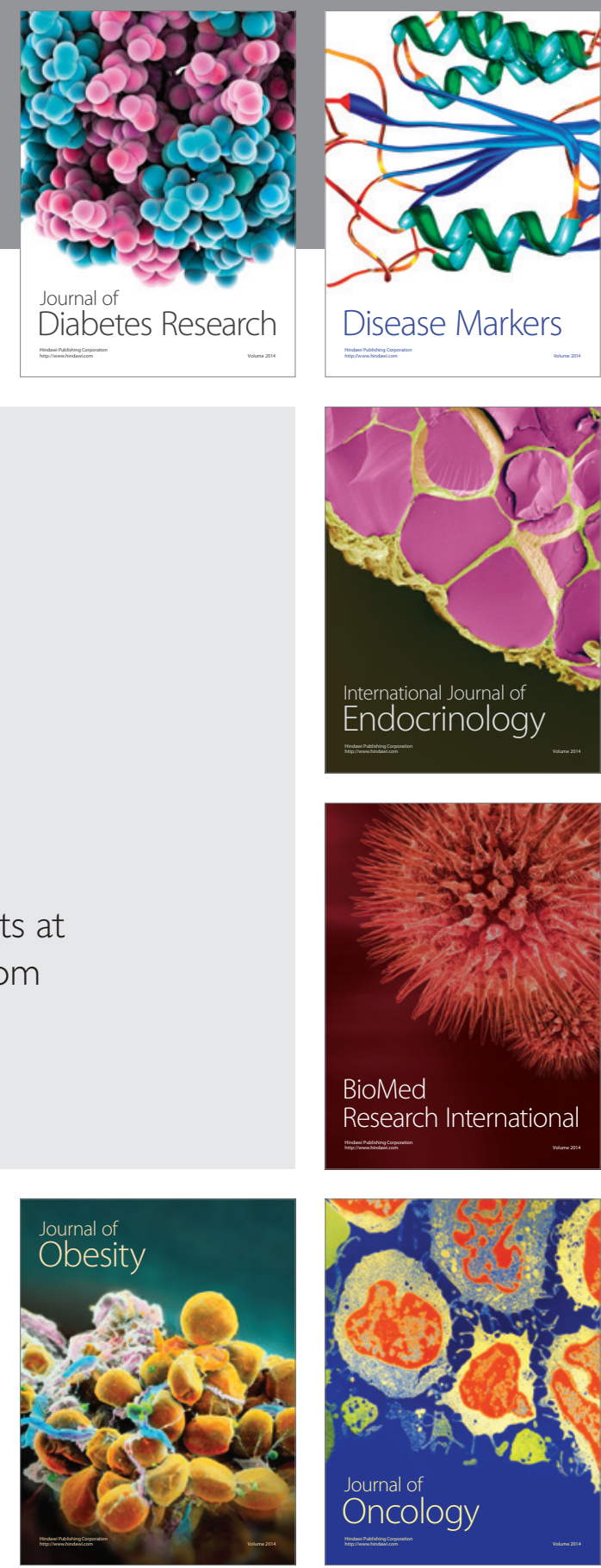

Disease Markers
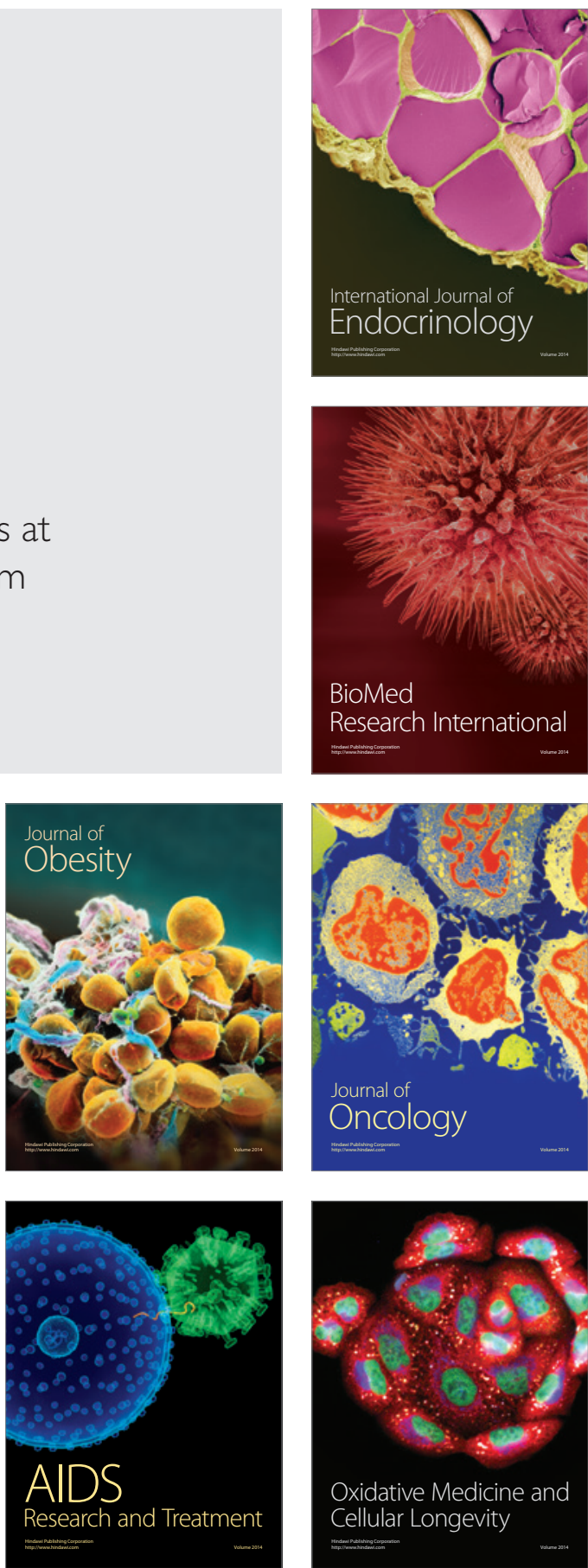